\title{
Un índice para evaluar la calidad del hábitat en la Franja Costera Sur del Río de la Plata y su vinculación con otros indicadores ambientales
}

\author{
Nora GÓMEZ ${ }^{\bowtie}$ \& JoAquín COCHERO \\ Instituto de Limnología Dr. R.A. Ringuelet, CONICET, CCT La Plata-Facultad de Ciencias Naturales y Museo (UNLP). \\ Buenos Aires, Argentina.
}

\begin{abstract}
Resumen. La valoración ambiental de las riberas es una tarea necesaria para conocer el estado ecológico de los cursos de agua, lo cual permite evaluar los méritos de eventuales experiencias de manejo, impactos antropogénicos o bien detectar problemas ambientales de forma temprana. En tal sentido, el objetivo de este estudio fue desarrollar una metodología para evaluar la calidad del hábitat de la Franja Costera Sur del Río de la Plata. La información obtenida de 21 sitios de muestreo, visitados entre los años 2005 y 2008 , fue resumida y simplificada en un índice, denominado Índice del Hábitat para el Río de la Plata (IHRPlata). Para obtener el índice se requiere evaluar cuatro descriptores: 1) sucesión espacial de la vegetación costera, 2) modificaciones costeras por introducción de infraestructuras, 3) ocurrencia de residuos en la línea de costa, y 4) indicadores biológicos de déficit de oxígeno. Según los valores del IHRPlata obtenidos, $32 \%$ de los casos analizados revelaron una calidad del hábitat muy buena, $20 \%$ buena, $24 \%$ moderada, $18 \%$ mala y $6 \%$ muy mala. Los valores del índice alcanzaron correlaciones significativas con parámetros relacionados con la calidad del agua y con indicadores de la calidad biótica, lo que fortalece su utilidad para evaluar la calidad del hábitat costero.
\end{abstract}

[Palabras clave: monitoreo, ribera, disturbios antropogénicos]

\begin{abstract}
Aвstract. An index to assess the habitat quality in Southern Coastal Fringe of the Río de la Plata and its relations with other environmental indicators: Evaluating the coastal environment is a necessary task to assess the ecological status of water bodies, which allows to determine the merits of management programs, anthropogenic impacts, or to detect early signs of environmental impacts. To this purpose, the objective of this research was to develop a methodology to assess the habitat quality in the Franja Costera Sur of the Río de la Plata. The information obtained from 21 sampling sites, visited from 2005 to 2008, is summarized and simplified in an index, named the Habitat Index for the Río de la Plata (IHRPlata). To obtain the index four separate descriptors are to be evaluated: 1) the spatial succession of the vegetation, 2) any man-introduced infrastructures in the coast, 3) the presence of litter and debris in the coastline, and 4) the visualization of biological indicators that imply oxygen deficit. According to the obtained values for the IHRPlata, 32\% of the analyzed cases revealed a very good habitat quality, $20 \%$ good, $24 \%$ moderate, $18 \%$ bad and $6 \%$ very bad habitat quality. The obtained values were also significantly correlated with parameters related to water quality and to biotic indicators, which strengthens its usefulness to evaluate the coastal habitat.
\end{abstract}

[Keywords: monitoring, riverside, anthropogenic disturbance]

\section{INTRODUCCIÓN}

Los disturbios generados por las intervenciones del hombre sobre los ecosistemas naturales costeros son profundos, lo cual ha conducido a una preocupación creciente por conocer su integridad ecológica para así adoptar medidas de gestión adecuadas para su conservación. La incertidumbre de las variables humanas y naturales que afectan el manejo integrado de zonas costeras enfatiza la necesidad de generar bases de datos ambientales sólidas que contribuyan a evaluar los méritos de eventuales experiencias de manejo, impactos ambientales o bien detectar eventuales problemas ambientales de manera temprana (FREPLATA 2005).

$\triangle$ Instituto de Limnología Dr. R.A. Ringuelet, CONICET, CCT La Plata-Facultad de Ciencias Naturales y Museo (UNLP). Buenos Aires, Argentina. nora@ilpla.edu.ar
En las últimas décadas ha surgido un interés particular en el ámbito científico (y en el de la gestión) por mejorar los conocimientos sobre los hábitats acuáticos; este interés es resultado de la necesidad de contar con información pertinente para el manejo y la restauración de esos hábitats. Jowet (1977) ha sugerido que el término genérico hábitat describe el entorno físico de plantas y animales, y por lo tanto el hábitat acuático puede ser definido como el conjunto de características físicas, químicas y biológicas locales que proporcionan un entorno para la biota. El hábitat es afectado por las características propias del cuerpo de agua y por la topografía circundante, que determinan el potencial de las comunidades biológicas (Aadland 1993). Este concepto es relevante ya

Recibido: 11 de junio de 2012, Fin de arbitraje: 20 de agosto, Última versión: 4 de diciembre, Aceptado: 14 de diciembre. 
que existen evidencias que demuestran que la cantidad y calidad de hábitats disponibles afectan la estructura y composición de las comunidades biológicas locales (Callow \& Petts 1994; Graham et al. 2000; Bortone 2005; Borja et al. 2009).

La valoración ambiental de las riberas es una tarea necesaria para conocer el estado ecológico de los cursos de agua. En tal sentido se han desarrollado distintas metodologías de evaluación considerando la vegetación riparia, peces, macroinvertebrados, características del cauce, etc., para sistemas fluviales del hemisferio norte (Karr 1999; Kaufmann et al. 1999; Barbour et al. 1999; Maddock 1999; Munné et al. 2003; Winward 2000; Raab \& Bayley 2012; González del Tánago et al. 2004). Muchas de estas propuestas requieren de metodologías de muestreo específicas, y demandan el empleo de dragas, tamices, redes, pesca eléctrica, etc., según la comunidad seleccionada como así también de un buen conocimiento taxonómico por parte de los potenciales usuario para su aplicación. En Argentina, los antecedentes existentes sobre la evaluación de las zonas ribereñas es exigua (Miserendino et al. 2010; Kutschker et al. 2009; Troitiño et al. 2010) y no se cuenta con antecedentes para ecosistema estuarinos del país, entre los que se encuentra el estuario del Río de la Plata. Con una superficie de $35000 \mathrm{~km}^{2}$, este estuario se caracteriza por ser somero, presentar 37\% de su extensión cubierta por agua dulce y una pequeña amplitud de marea $(\sim 1 \mathrm{~m})$ que puede ser superada durante los fenómenos meteorológicos conocidos como sudestadas. Su morfología y dinámica determinan dos zonas: una interna $\left(13000 \mathrm{~km}^{2}\right.$ cubiertos con agua dulce y profundidades entre 1 y $5 \mathrm{~m})$ y otra externa $\left(22000 \mathrm{~km}^{2} \mathrm{de}\right.$ superficie y profundidades entre 5 y $25 \mathrm{~m}$, con características mixohalinas). El grado elevado de urbanización e industrialización que se concentra en la zona interna del estuario del Río de la Plata, especialmente a lo largo de la costa argentina, genera el ingreso de contaminantes que representan una amenaza tanto para la biota como para la salud humana. Entre estos contaminantes podemos mencionar nutrientes, materia orgánica, metales pesados, plaguicidas, hidrocarburos, sólidos en suspensión y agentes patógenos. Además, los dragados y la modificación del régimen hidrológico de los humedales costeros, entre otras intervenciones humanas, han alterado la morfología de la costa interfiriendo tanto la integridad del hábitat físico como los procesos naturales (FREPLATA 2005).
La integridad ecológica consiste en un sistema biofísico en el cual prevalece una composición de especies y una organización funcional comparable a la de los ecosistemas naturales de una determinada región ecológica (Karr 1991). Por lo tanto, resulta fundamental contar con indicadores para la costa del Río de la Plata que permitan la evaluación del hábitat para así contribuir a determinar la integridad ecológica de la zona costera. La existencia de este conocimiento es primordial para poder estimar la capacidad del sistema a resistir y recuperarse de las perturbaciones impuestas por los procesos naturales del medio ambiente, así como de los trastornos inducidos por el hombre. En este trabajo se propone una metodología para evaluar la calidad del hábitat costero de la Franja Costera Sur del Río de la Plata. La información obtenida es resumida y simplificada en un índice, denominado Índice del Hábitat para el Río de la Plata (IHRPlata), cuya finalidad es la de transferir la información obtenida de manera clara y que pueda ser interpretada por el público, los medios de comunicación, usuarios de los recursos y los tomadores de decisiones. Para ello se definieron descriptores de la calidad ambiental de la zona costera, de aplicación sencilla. Estos descriptores incluyen la evaluación de la sucesión espacial de los principales tipos de vegetación ribereña, la presencia de infraestructuras introducidas por el hombre que modifican la dinámica costera, la ocurrencia de basura acumulada en la línea de costa y la detección de indicadores bióticos que delatan el déficit de oxígeno. También se estableció la relación entre los valores del IHRPlata obtenidos para el área de estudio con los porcentajes de uso del suelo cercanos a la costa, con indicadores de la calidad del agua y de la integridad biótica.

Consideramos que si bien la zona en estudio está sujeta a procesos naturales que modifican las características del hábitat físico (e.g., erosión, acumulación) es probable que estos procesos se acentúen por la frecuencia y severidad de las diferentes intervenciones humanas. La combinación de los descriptores antes mencionados en un índice dinámico pretende brindar información sobre el estado del hábitat costero para albergar la biota, y también proveer al usuario que revisita los sitios afectados por intervenciones humanas (e.g., urbanizaciones costeras, obras de saneamiento, expansión de áreas recreativas, entre otras) con una herramienta que evalué cambios a lo largo del tiempo. 


\section{Materiales y Métodos}

\section{Área de estudio}

Elárea de estudio se situó entre la desembocadura del Río Luján y la localidad de Punta Indio. Se establecieron 21 sitios de muestreo distribuidos a lo largo de $170 \mathrm{~km}$ de costa (Figura 1), ubicados en el sector intermareal del área de agua dulce del estuario (salinidad inferior a los 0.5 Unidades Prácticas de Salinidad). En la Tabla 1 se detalla la localización de los sitios de muestreo y las barreras físicas introducidas por el hombre. Los sitios fueron georeferenciados y visitados al menos en tres oportunidades entre los años 2005 y 2008. Las principales características físico-químicas del agua figuran en la Tabla Suplementaria 1 y fueron extraídas de Gómez et al. $(2009,2012)$.
Caracterización de los sitios estudiados y expresión del Indice del hábitat (IHRPlata)

Para la evaluación del uso del suelo cercano a la costa se determinó una franja de $10 \mathrm{~km}$ de ancho por $170 \mathrm{~km}$ de largo que incluyó todos los sitios muestreados. Se obtuvieron mapas de uso del suelo a una escala 1:30000 (Figura 1) a partir de imágenes satelitales con un sistema de información geográfica (Quantum GIS 1.6). Los usos del suelo fueron agrupados en: áreas rurales o recreativas (reúne núcleos urbanos con menos de 2500 habitantes, se practica principalmente ganadería extensiva $\mathrm{y}$ actividades recreativas como pesca, deportes acuáticos, baño, etc.), áreas suburbanas con baja a moderada influencia humana (reúne núcleos urbanos >2500-20000 habitantes, con actividad industrial baja), y áreas urbanas que aglutinan

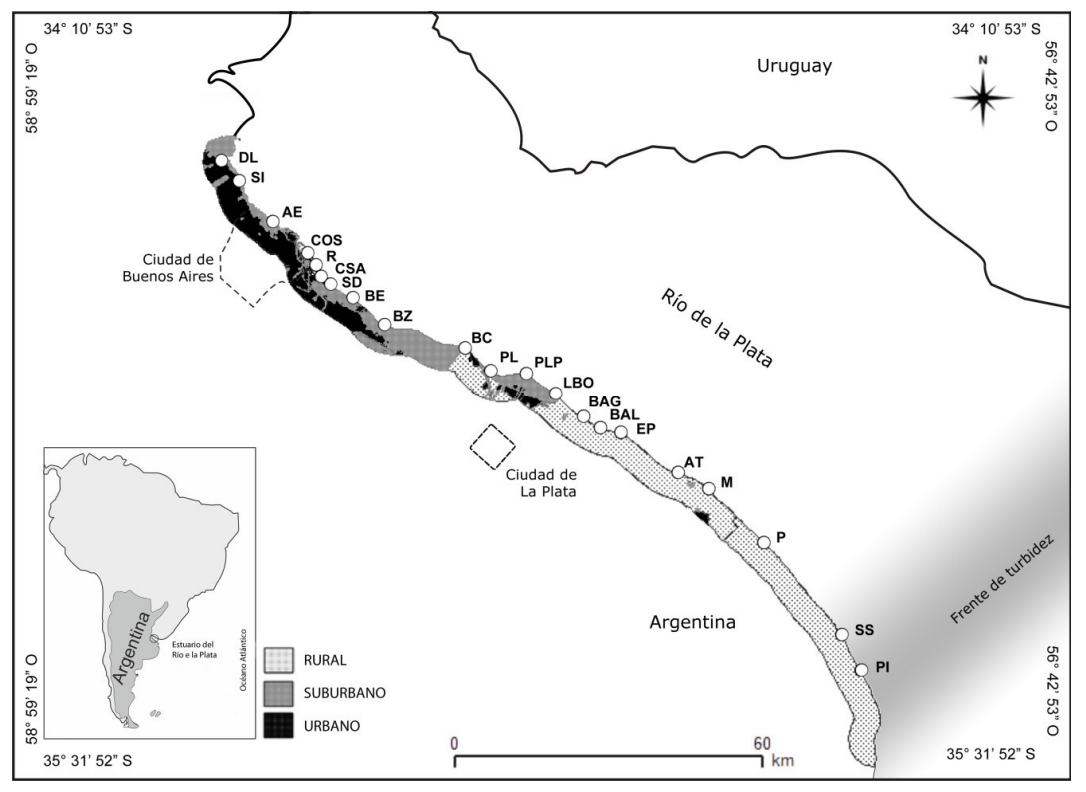

Figura 1. Mapa del área de estudio con los usos del suelo en la zona aledaña a la costa y la localización de los sitios de estudio (descripción en Tabla 1).

Figure 1. Map of the study area with the main land uses in the coast and the location of the sampling sites (description in Table 1).

Figura 2. Diagrama de las escalas utilizadas para la evaluación de la calidad del hábitat en la zona costera del Río de la Plata.

Figure 2. Diagram of the scales used to measure the different indicators used to assess the habitat quality in the coastal zone of the Río de la Plata.

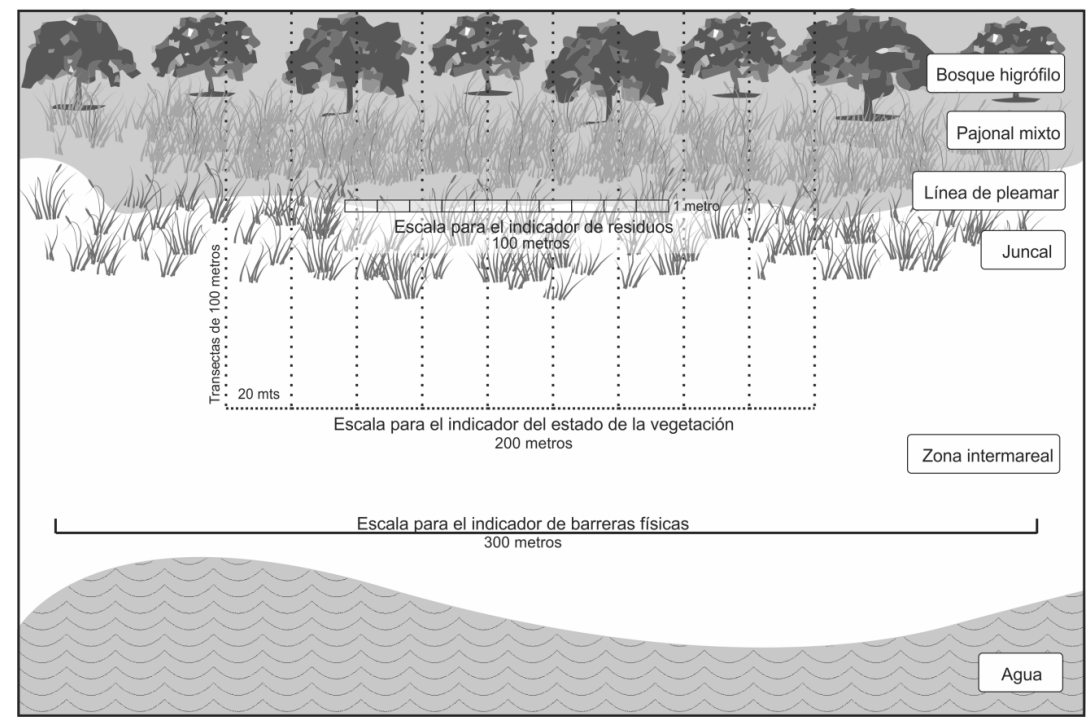


Tabla 1. Ubicación de los sitios de muestreo, porcentajes del uso del suelo y modificaciones costeras introducidas por el hombre.

Table 1. Coordinates of the sampling sites, the percentage of the land uses and the man-made coastal modifications.

\begin{tabular}{|c|c|c|c|c|c|}
\hline Acrónimo & Sitios & Urbano & Suburbano & Rural & Barreras físicas \\
\hline DL & Desembocadura de Luján & 61.7 & 38.3 & 0 & $\begin{array}{l}\text { Estructuras temporarias } \\
\text { y escolleras }\end{array}$ \\
\hline SI & San Isidro & 85.7 & 14.3 & 0 & Estructuras temporarias rellenos y escolleras \\
\hline $\mathrm{AE}$ & Aeroparque & 75.0 & 25.0 & 0 & Murallas \\
\hline $\cos$ & Costanera Sur & 86.8 & 0.3 & 12.9 & Estructuras temporarias y rellenos \\
\hline $\mathrm{R}$ & Matanza -Riachuelo & 100 & 0 & 0 & Murallas \\
\hline CSA & Canal Sarandí & 61.2 & 38.3 & 0.6 & Sin modificaciones \\
\hline SD & Canal Santo Domingo & 61.2 & 38.3 & 0.6 & $\begin{array}{l}\text { Estructuras temporarias } \\
\text { y rellenos }\end{array}$ \\
\hline $\mathrm{BE}$ & Bernal & 60.8 & 38.7 & 0.5 & Estructuras temporarias \\
\hline $\mathrm{BZ}$ & Berazategui & 39.3 & 60.7 & 0 & Estructuras temporarias y rellenos \\
\hline $\mathrm{BC}$ & Boca Cerrada & 7.6 & 42.1 & 50.2 & Murallas \\
\hline PL & Punta Lara & 19.0 & 11.6 & 69.4 & Estructuras temporarias \\
\hline PLP & Puerto La Plata & 31.7 & 68.1 & 0.2 & Murallas \\
\hline LBO & Los Borrachos & 13.3 & 38.9 & 47.9 & Estructuras temporarias y rellenos \\
\hline BAG & Bagliardi & 1.2 & 8.3 & 90.5 & Estructuras temporarias y rellenos \\
\hline BAL & Balandra & 0 & 0 & 100 & Estructuras temporarias \\
\hline EP & El Pino & 0 & 0 & 100 & Sin modificaciones \\
\hline AT & Atalaya & 2.0 & 0 & 98.0 & Estructuras temporarias y escolleras \\
\hline M & Magdalena & 3.9 & 0 & 96.1 & Estructuras temporarias \\
\hline $\mathrm{P}$ & Pearson & 0 & 0 & 100 & Sin modificaciones \\
\hline SS & Sarandí Sur & 0 & 0 & 100 & Sin modificaciones \\
\hline PI & Punta Indio & 0 & 0 & 100 & Estructuras temporarias \\
\hline
\end{tabular}

zonas pobladas densamente (núcleos urbanos $>50000$ habitantes, megalópolis como la ciudad de Buenos Aires y La Plata, con actividad industrial diversificada e intensa).

Tabla 2. Valores que pueden asumir el indicador de sucesión espacial de la vegetación costera (SEV) (según el número de transectas donde se visualice la sucesión de juncales, pajonales mixtos y bosque higrófilo) y el indicador de ocurrencia de residuos ajenos a la costa (OR) (según el número de parcelas donde se detectan los residuos).

Table 2. Values that can be assumed by the spatial succession indicator of the coastal vegetation (SEV) and by the debries occurrence (OR) (see details in legend in Spanish).

\begin{tabular}{ccc}
\hline $\begin{array}{c}\text { Sucesión espacial } \\
\text { de la vegetación } \\
\text { costera (SEV) } \\
\mathrm{N}^{\circ} \text { de transectas }\end{array}$ & $\begin{array}{c}\text { Ocurrencia de } \\
\text { residuos ajenos a } \\
\text { la costa (OR) } \\
\mathrm{N}^{\circ} \text { de parcelas }\end{array}$ & $\begin{array}{c}\text { Valor indicador } \\
\text { de SEV y OR }\end{array}$ \\
\hline 10 & 0 & 10 \\
9 & 1 & 9 \\
8 & 2 & 8 \\
7 & 3 & 7 \\
6 & 4 & 6 \\
5 & 5 & 5 \\
4 & 6 & 4 \\
3 & 7 & 2 \\
2 & 8 & 1 \\
1 & 9 & 0 \\
0 & 10 & \\
\hline
\end{tabular}

En la costa del Río de la Plata se reconoce entre el ecosistema acuático y el terrestre una sucesión de diferentes tipos de vegetación (SEV), determinados por las formas de vida, (Hurrell 2004). En los sitios del área de estudio poco afectados por la intervención humana, es posible observar juncales dominados por Schoenoplectus californicus (Mey) Steud, que son sucedidos por pajonales mixtos compuestos principalmente por Cortaderia selloana (Schult. \& Schult. f.) Asch. \& Graebn., Scirpus giganteus Kunth, Zizaniopsis bonariensis (Balansa \& Poitr.) Speg, entre otras y por el bosque higrófilo conformado principalmente por Salix humboldtiana Willd y Erythrina crista-galli L. (Figura 2). Para evaluar la continuidad de esta sucesión se consideró un tramo de $200 \mathrm{~m}$ de largo y $100 \mathrm{~m}$ de ancho. En cada sitio de muestreo se establecieron 10 transectas perpendiculares a la línea de costa equidistante cada $20 \mathrm{~m}$ (Figura 2). Se consideró un estado óptimo de este indicador cuando las 10 transectas contaban con la presencia de la sucesión antes detallada, y la peor condición correspondió a la ausencia de aquella en todas las transectas; situaciones intermedias adquieren valores que son detallados en la Tabla 2.

Para evaluar las modificaciones en las características del hábitat (MC), como consecuencia de las alteraciones que genera la instalación de infraestructuras en la costa, se analizó un tramo de $300 \mathrm{~m}$ (Figura 2). En este tramo se evaluó la presencia de estructuras temporarias (e.g., las destinadas a fines recreativos) y permanentes (e.g., 
escolleras, rellenos costeros, muelles y murallas). Este descriptor captura información vinculada con la disminución del grado de conectividad entre el ecosistema terrestre y acuático. La mejor condición para este indicador fue la ausencia de modificaciones en la costa y la peor la presencia de murallas de contención paralelas a la línea de costa, que desvincula totalmente el ecosistema acuático con el terrestre; las situaciones intermedias son detalladas en la Tabla 3.

Se realizó una estimación cualitativa de la presencia de residuos en la costa (OR), para lo cual se estableció un tramo de $100 \mathrm{~m}$ de largo por $1 \mathrm{~m}$ de ancho, ubicado en la línea de pleamar, que fue fraccionado cada $10 \mathrm{~m}$ (Figura 2). En estas parcelas se registró la presencia de residuos tales como plásticos, vidrio, metal, papel, tela, goma, desechos orgánicos domésticos y de higiene personal, entre otros. La condición óptima de este indicador es alcanzada cuando en ninguna de las parcelas se observan elementos ajenos a la costa y la peor cuando en las 10 parcelas se encuentra algunos de aquellos; las situaciones intermedias son detalladas en la Tabla 2.

Como indicador del déficit de oxígeno (DO) se recurrió a la observación directa del desarrollo de matas de bacterias filamentosas, que están conformadas principalmente por bacterias saprófitas anaeróbicas o facultativas tales como las pertenecientes a los géneros Beggiatoa, Leptothrix, Sphaerotilus, entre otras. La visualización de este indicador es sencilla ya que se identifica a través de la observación en campo de matas gelatinosas y blanquecinas que proliferan sobre sustratos naturales como sedimento, plantas o sobre objetos ajenos a la costa. Su presencia indica condiciones severas de contaminación, que conllevan a disminuir las concentraciones del oxígeno disuelto debido al consumo por parte de los organismos aeróbicos para descomponer la materia orgánica. A este indicador se le asignó un valor de 10 si está ausente y de 0 si está presente.
Tabla 3. Valores que puede asumir el indicador de modificaciones costeras (MC) introducidas por el hombre.

Table 3. Values that can be assumed by the indicator of man-made coastal modifications (MC).

\begin{tabular}{lc}
\hline Barreras físicas & $\begin{array}{c}\text { Valor del } \\
\text { indicador }\end{array}$ \\
\hline Sin modificaciones & 10 \\
Estructuras temporarias & 7.5 \\
Estructuras temporarias, rellenos & 5 \\
$\begin{array}{l}\text { Estructuras temporarias, rellenos, } \\
\text { escolleras y/o muelles }\end{array}$ & 2.5 \\
Murallas & 0 \\
\hline
\end{tabular}

Para evaluar el estado del hábitat (IHRPlata) se promediaron los cuatro indicadores antes descriptos (SEV, OR, MC y DO), por lo que el IHRPlata puede asumir valores entre 0 y 10 . Se establecieron 5 calidades del hábitat, son detallados en la Tabla 4.

\section{Análisis estadístico}

Se emplearon correlaciones de Pearson para explorar la relación entre los valores del IHRPlata con los porcentajes de uso del suelo cercanos a la costa, con indicadores de la calidad del agua

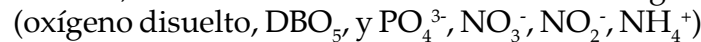
y con los descriptores de la calidad biótica. Para estos descriptores de la calidad biótica se recurrió a los ensambles de macroinvertebrados bentónicos considerando la riqueza de taxa, la diversidad $\mathrm{H}^{\prime}$ (Shannon \& Weaver 1949), el Índice de Macroinvertebrados para Ríos Pampeanos (IMRP; Rodrigues Capítulo et al. 2001). También se realizó una correlación con el índice de integridad biótica diseñado para la costa del Río de la Plata (IBIRP; Gómez et al. 2012), que considera indicadores de eutrofización y contaminación, empleando descriptores del plancton y del bentos de la zona intermareal. Los detalles metodológicos sobre los

Tabla 4. Valores de IHRPlata, categorías y su significado.

Table 4. Values that the IHRPlata can assume, its categories and interpretation.

\begin{tabular}{|c|c|c|}
\hline $\begin{array}{l}\text { Valor del } \\
\text { IHRPlata }\end{array}$ & Categoría & Principales características del hábitat \\
\hline$>8-10$ & Muy buena & $\begin{array}{l}\text { Sucesión espacial de los tipos de vegetación ribereña no alterada, ausencia o muy baja } \\
\text { ocurrencia de residuos, ausencia de indicadores de déficit de oxígeno disuelto, muy buena } \\
\text { conectividad entre el ecosistema acuático y terrestre. }\end{array}$ \\
\hline$>6-8$ & Buena & $\begin{array}{l}\text { Sucesión espacial de los tipos de vegetación ribereña ligeramente modificada, baja } \\
\text { ocurrencia de residuos, ausencia de indicadores de déficit de oxígeno disuelto, buena } \\
\text { conectividad entre el ecosistema acuático y terrestre. }\end{array}$ \\
\hline$>4-6$ & Moderada & $\begin{array}{l}\text { Sucesión espacial de los tipos de vegetación ribereña se halla modificada, moderada cantidad } \\
\text { de residuos, ausencia de indicadores de déficit de oxígeno disuelto, conectividad aceptable } \\
\text { entre el ecosistema acuático y terrestre. }\end{array}$ \\
\hline $2-4$ & Mala & $\begin{array}{l}\text { Marcada modificación de la sucesión espacial de los tipos de vegetación ribereña, moderada } \\
\text { a alta ocurrencia de residuos, presencia de indicadores de déficit de oxígeno disuelto, } \\
\text { conectividad entre el ecosistema acuático y terrestre disminuida. }\end{array}$ \\
\hline
\end{tabular}


análisis fisicoquímicos y biológicos figuran en Gómez et al. (2009) y Gómez et al. (2012).

\section{Resultados}

El 32\% de los casos analizados reveló, según el IHRPlata, una calidad del hábitat muy buena, $20 \%$ buena, $24 \%$ moderada, $18 \%$ mala y $6 \%$ muy mala. Los valores más altos del índice se ubicaron en el sector más distal del área de estudio, en particular en los sitio EP y SS (Figura 3). Los valores más bajos del IHRPlata se localizaron en el sector comprendido entre la desembocadura del Río Luján y la ciudad de La Plata, y correspondieron a los sitios R y PLP los valores de 0 .

Las correlaciones entre los valores del IHRPlata y los porcentajes de usos del suelo demostraronunarelaciónnegativasignificativa con los usos urbano y suburbano, y positiva con el predominio del uso rural (Tabla 5). Por otra parte el IHRPlata se correlacionó de manera significativa con indicadores de la calidad del agua, obteniendo una relación positiva con el incremento del oxígeno disuelto y negativa con el aumento en la concentración de nutrientes y la demanda biológica de oxígeno (Tabla 5). Asimismo, el índice se correlacionó significativamente con descriptores de los ensambles de macroinvertebrados y con el IBIRP relacionados con la calidad biótica de la costa.(Tabla 5).

El análisis detallado de los cuatro descriptores empleados para calcular el IHRPlata (Figura
Tabla 5. Coeficiente de correlación de Pearson significativos $\left({ }^{* *} P<0.001,{ }^{*} P<0.05, \mathrm{n}=106\right)$ entre el IHRPlata, el porcentaje de usos del suelo, indicadores de la calidad del agua e indicadores de la calidad biológica.

Table 5. Significant Pearsons correlation coefficients $\left({ }^{* *} P<0.001,{ }^{*} P<0.05, \mathrm{n}=106\right)$ between the IHRPlata, the land use percentages the water and biological quality indicators.

\begin{tabular}{llc}
\hline & & IHRPlata \\
\hline Usos del & Urbano & $r-0.71^{* *}$ \\
suelo & Suburbano & $r-0.76^{* *}$ \\
& Rural & $r 0.79^{* *}$ \\
& Oxígeno disuelto & $r 0.38^{* *}$ \\
& $\mathrm{DBO}_{5}$ & $r-0.43^{* *}$ \\
Calidad & $\mathrm{PO}_{4}^{3-}$ & $r-0.42^{* *}$ \\
del agua & $\mathrm{NO}_{3}^{-}$ & $r-0.34^{* *}$ \\
& $\mathrm{NO}_{2}^{-}$ & $r-0.65^{* *}$ \\
& $\mathrm{NH}_{4}{ }^{-}$ & $r-0.34^{* *}$ \\
& $\mathrm{Riqueza}^{-}(\mathrm{R})$ & $r 0.35^{* *}$ \\
& Diversidad (H') & $r 0.23^{*}$ \\
Calidad & $\mathrm{IMRP}^{-}$(Índice de & \\
biológica & Macroinvertebrados para Ríos & $r 0.45^{* *}$ \\
& Pampeanos) & \\
& IBIRP (Índice de Integridad & $r 0.39^{* *}$ \\
\hline & Biótica para el Río de la Plata) & \\
\hline
\end{tabular}

4) permitió identificar que los disturbios que provocan la modificación de la costa por introducción de infraestructuras y la ocurrencia de basura influyeron de forma marcada en la disminución del valor del índice en $50 \%$ de los sitios analizados. En tanto en 38\% y $33 \%$ de los sitios analizados, la destrucción de la vegetación costera y el déficit $\square$ Promedio $\square$ Error estándar I Desvío estándar

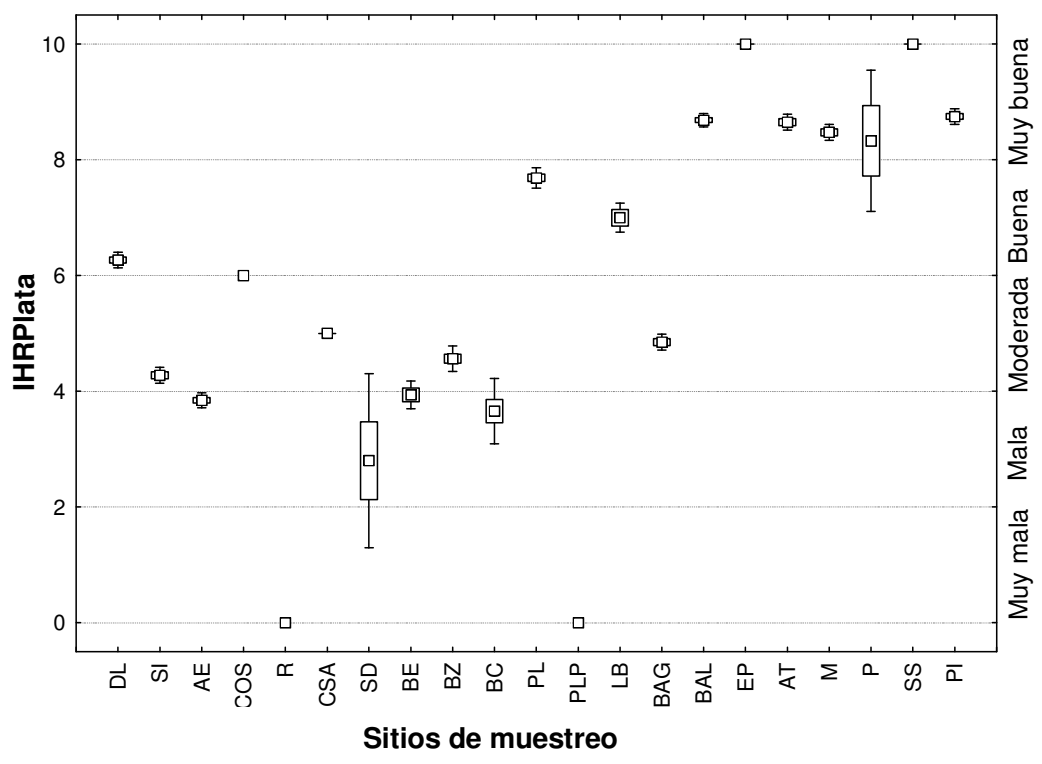

Figura 3. Promedio, error y desvío estándar de los valores del IHRPlata y la categoría correspondiente a la calidad del hábitat obtenida para los distintos sitios de muestreo.

Figure 3. Mean, standard error and standard deviation of the IHRPlata values with their corresponding categories for the habitat quality obtained for the sampled sites. 


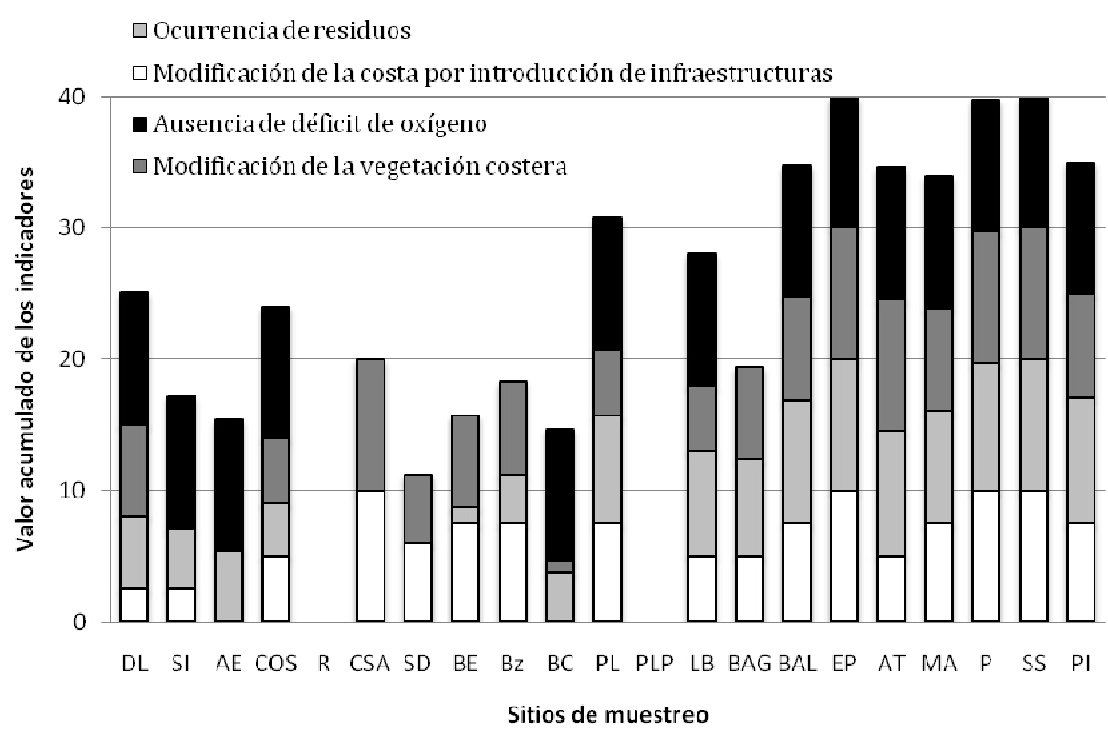

Figura 4. Valores promedio acumulados para los descriptores empleados para calcular el IHRPlata en los distintos sitios de muestreo [ $n=3$ (R, PLP, COS), $\mathrm{n}=7$ (DL), $\mathrm{n}=5$ (para los restantes sitios de muestreo)].

Figure 4. Mean accumulated values for each indicator used to calculate the IHRPlata index in the different sampling sites $[n=3(R$, PLP, COS), $\mathrm{n}=7$ (DL), $\mathrm{n}=5$ (for all remaining sites)]. de oxígeno intervinieron -respectivamente- en la disminución de los valores del IHRPlata.

\section{DisCUSIÓN}

Los cuatro indicadores seleccionados para la elaboración del IHRPlata evidencian algunas de las problemáticas más frecuentes que surgen como consecuencia de las amenazas de las actividades realizadas por el hombre en al ámbito costero. Entre ellas se reconoce la contaminación orgánica e inorgánica, acompañada del aporte de basura domiciliaria, que es transportada por el agua o depositada directamente en la costa. Ésta constituye una barrera física para el libre intercambio entre el medio acuático y el terrestre debido a que se enreda entre los tallos y rizomas de la vegetación, contribuyendo además a disminuir la estética natural de la ribera. Asimismo, los cambios en la configuración de la línea de costa debidos a los diferentes tipos de infraestructuras introducidas por el hombre constituyen otro factor que de manera frecuente afecta la dinámica costera. Estas alteraciones modifican las condiciones de erosión y/o deposición en diferentes lugares de la costa, y favorecen la erosión de áreas que naturalmente tenderían a experimentar acumulación, y a generar acumulación en áreas naturalmente expuestas a la erosión (Kokot \& Guerrieri 2005; Kokot 2005; López \& Marcomini 2004). Por ejemplo, los rellenos artificiales, introducidos en sectores de la costa a partir de mediados del siglo XIX, han incrementado la superficie de la planicie del estuario, ganando terrenos al Río de la Plata (Atlas Ambiental de Buenos Aires
2010). Además, los escombros depositados en la costa, remanentes de las actividades productivas provenientes de las excavaciones para la construcción y renovación de infraestructuras de las urbes, sufren procesos erosivos que modifican la composición y granulometría natural del sedimento costero. Así, los sedimentos de la zona intermareal, constituidos por arenas finas y muy finas, fueron reemplazados por otros más gruesos, que modificaron las condiciones del hábitat para la biota asociada al bentos (Gómez et al. 2009).

La modificación de la vegetación ribereña, como consecuencia de la actividad humana, es otro factor clave en la ecología costera. La preservación de la vegetación riparia es fundamental, ya que las plantas acuáticas junto al sustrato en el que se asientan sirven de hábitat para numerosas especies, proporcionándoles alimento y refugio y favoreciendo además la estabilización de los sedimentos (Gonzáles del Tánago \& Jalón 2004). Por lo tanto la destrucción de la vegetación constituye un síntoma de deterioro ambiental, que conlleva a la reducción de los hábitats para distintos niveles tróficos.

La integración de los descriptores requeridos para calcular el IHRPlata contempló algunas de las consecuencias que acarrean los disturbios antropogénicos sobre la costa, permitiendo identificar sitios con distintas calidades del hábitat en la Franja Costera Sur del Río de la Plata. Los valores más bajos del IHRPlata se localizaron en cercanías de la ciudad de Buenos Aires y La Plata. En estos sitios se advierte una mala calidad del agua, 
lo que contribuye a deteriorar la condición de los sedimentos intermareales al enriquecerlos con materia orgánica (Gómez et al. 2009; Gómez et al. 2012) y con una carga variada de otros contaminantes que atentan contra la biodiversidad (César 2000; FREPLATA 2005; INA-LHA 2011). La consecuencia primaria en los sitios más afectados por esta problemática es la disminución en la concentración de oxígeno disuelto, cuya manifestación visual se evidencia por la proliferación de indicadores de condiciones polisapróbicas, tales como el desarrollo de bacterias filamentosas. En particular, estas bacterias crecen en sitios enriquecidos con materia orgánica, donde el oxígeno disminuye de forma abrupta hasta originar situaciones de anaerobiosis, y generan subproductos orgánicos e inorgánicos como $\mathrm{CH}_{4}, \mathrm{CO}_{2} \mathrm{y} \mathrm{S}_{2} \mathrm{H}$. Algunos de estos compuestos confieren mal olor al ambiente (Sladecek 1973; Branco 1984; Atlas \& Bartha 2002). Así mismo, el enriquecimiento con materia orgánica es acompañado por concentraciones altas de nutrientes que promueven procesos de eutrofización en el agua y en los sedimentos de la costa (Gómez \& Bauer 2000; Gómez et al. 2002; Gómez et al. 2009; Licursi et al. 2010; Gómez et al. 2012). También las modificaciones profundas de la línea de costa observadas en los sitios con bajos valores de IHRPlata suelen favorecer los procesos de acumulación de los contaminantes y basura, a los cuales se le añade la destrucción de la vegetación costera. Esta destrucción es causada principalmente por el desmonte para establecer rellenos costeros, áreas de recepción de residuos domiciliarios, por aumentar las vías de acceso a la costa y por el avance de la urbanización.

Los sitios que obtuvieron la mejor condición del hábitat se localizaron más al sur, alejados de los principales conglomerados urbanos, donde el uso del suelo es mayormente rural (i.e., la ganadería extensiva). Si bien en estos sitios de muestreo la energía del estuario se hace manifiesta sobre la línea costera, por un mayor efecto de la erosión mecánica y exposición a las sudestadas, la ribera conserva una condición óptima para la mayoría de los indicadores evaluados en el cálculo del índice. El índice presentó correlaciones significativas con indicadores de la calidad del agua, de la calidad biótica relacionados con los ensambles de invertebrados y con el índice de integridad biótica IBIRP. Esto fortalece su utilidad para evaluar la calidad del hábitat costero. Por otra parte, la combinación entre la información aportada por este índice, la provista por la calidad del agua y el IBIRP constituye un avance significativo para definir la integridad ecológica de la Franja Costera Sur.

Cabe destacar que a diferencia de otros índices que evalúan la calidad del hábitat físico a partir del empleo de las comunidades de peces o de macroinvertebrados (Meng et al. 2002; Díaz et al. 2004; Harrison \& Whitfield 2006), el IHRPlata recurre a indicadores que no requieren de la complejidad de los muestreos y análisis que demandan el empleo aquellas comunidades. Por otra parte si bien el IHRPlata fue diseñado a partir de información procedente del sector fluvial del estuario del Río de la Plata, se espera que con las adaptaciones pertinentes referidas particularmente a los patrones de la sucesión espacial de la vegetación riparia, pueda ser empleado en la evaluación de la calidad del hábitat de otros estuarios y cursos de agua antropizados.

Agradecimientos: Este estudio fue financiado por los proyectos PIP- CONICET 1556 y PICT 32077. Agradecemos a los integrantes de los laboratorios de Plancton y Biofilms y Bentos del ILPLA por el apoyo brindado en el trabajo en campo. Contribución Científica $\mathrm{N}^{\circ} 918$ del Instituto de Limnología "Dr. Raúl A. Ringuelet" (CONICET-UNLP). También agradecemos a los revisores que con sus correcciones y sugerencias contribuyeron a mejorar la versión final de este artículo.

\section{BibLIOGRAFíA}

AAdLAND, LP. 1993. Stream habitat types: their fish assemblages and relationships to flow. N. Am. J. Fish. Manage., 13:790-806.

Atlas Ambiental de Buenos Aires. www.atlasdebuenos aires.gov.ar.

AtLAS, RM \& R BARTHA. 2002. Ecología microbiana y microbiología ambiental. Pearson Educación S.A. Madrid, España. Pp. 677.

Barbour, MT; J Gerritsen; BD Zinder \& JB Stribling. 1999. Rapid bioassessment protocols for use in streams and wadeable rivers: Periphyton, benthic macroinvertebrates and fish. 2nd edition, EPA 841-B-99-002. Washington, DC.

Borja, A; J Bald; J Franco; J Larreta; I Muxica; et al. 2009. Using multiple ecosystem components, in assessing ecological status in Spanish (Basque Country) Atlantic marine waters. Mar. Pollut. Bull., 59:54-64.

Bortone, SA. 2005. Estuarine indicators. CRC Press, Boca Raton, Florida. Pp. 507.

BranCo, S. 1984. Limnología sanitaria. Estudio de la polución de las aguas continentales. OEA. Washington. Pp. 120.

Callow, P \& GE PetTs (eds.). 1994. The rivers handbook. Vol. 2. Blackwell Scientific, Oxford.

César, I; CS Ocón; AC Paggi; A Rodrigues Capítulo; F SPACCESI; ET AL. 2000. Diversidad de invertebrados bentónicos del Río de la Plata. Pp. 27-71 en: Gómez, N \& A Rodrigues Capítulo (eds.). Biodiversidad en la Franja Costera Sur del Río de la Plata. Fitoplancton, zoobentos, peces de la zona portuaria de la Ciudad de Bs. As. Biol. Acuát. 19:27-64.

Díaz, RJ; M Solan \& RM Valente. 2004. A review 
of approaches for classifying benthic habitats and evaluating habitat quality. J. Environ. Manage., 73: 165-181.

Freplata. 2005. Análisis Diagnóstico Transfronterizo del Río de la Plata y su Frente Marítimo. Proyecto "Protección Ambiental del Río de la Plata y su Frente Marítimo: Prevención y Control de la Contaminación y Restauración de Hábitats". Documento Técnico. Proyecto PNUD/GEF RLA/99/G31. Montevideo. Uruguay.

Gómez, N \& D BAuer. 2000. Diversidad fitoplanctónica en la Franja Costera Sur del Río de la Plata. Biol. Acuát., 19:7-26.

GÓmEZ, N; M Licursi; DE BAUER; ES AMBrosio; A RODRIGUeS CApítulo. 2012. Assessment of biotic integrity of the coastal freshwater tidal zone of a temperate estuary of South America through multiple indicators. Estuar. Coast., 35:1328-1339.

Gómez, N; M Licursi \& J Cochero. 2009. Seasonal and spatial distribution of the microbenthic communities of the Rio de la Plata estuary (Argentina) and possible environmental controls. Mar. Pollut. Bull., 58:878-887.

GÓMEz, N; DE BAuER; M Licursi \& PR HuAdDE. 2002. Planktonic and periphytic coastal algae of the Río de la Plata, Argentina. Verh. Internat. Verein. Limnol., 28:250-253.

Gonzáles del Tánago, M \& D García de Jalón. 2004. Recuperación de espacios degradados: cursos de agua desnaturalizados. Pp. 465-486 en: Gómez Orea, D. Restauración de Espacios Degradados. Cursos de agua desnaturalizados. Mundi-Prensa, Madrid, España.

González del Tánago, M; D García de Jalón; F Lara \& R GARILLETI. 2006. Índice RQI para la valoración de las riberas fluviales en el contexto de la directiva marco del agua. Ingeniería Civil, 143:97-108.

Graham, J; NS Barret; DJ Graddon \& PR Last. 2000. The conservation significance of estuaries: a classification of Tasmanian estuaries using ecological, physical and demographic attributes as a case study. Biol. Conserv., 92:383 -397.

HARrison, TD \& AK Whitfield. 2006. Application of a multimetric fish index to assess the environmental condition of South African estuaries. Estuar. Coasts, 29: 1108-1120.

Hurrell, JA. 2004. Plantas de la costa. Buenos Aires. L.O.L.A. $1^{\text {a }}$ ed. Pp. 200.

INA-LHA. 2011. Evaluación de la Calidad del Agua en la Franja Costera Sur del Río de la Plata mediante Modelación Matemática. Proyecto INA 1.207 Informe LHA 02-1.20711. Pp. 159.

JowET, IG (ed.). 1977. Instream flow methods: a comparison of approaches. Regul. River., 13:115-127.

KARR, JR. 1991. Biological integrity: a long-neglected aspect of water resource management. Ecol. Appl., 1:66-84.

KARR, JR. 1999. Defining and measuring river health. Freshwater Biol., 41:221-234.
Kaufmann, PR; P Levine; EG Robison; C Seeliger \& DV PeCK. 1999. Quantifying Physical Habitat in Wadeable Streams. EPA/620/R-99/003. U.S. Environmental Protection Agency, Washington, DC.

Кокот, R \& J GuerRieri. 2005. Evolución de la línea de costa Argentina, $2^{a}$ Comunicación de Cambio Climático "vulnerabilidad de la zona costera". Fundación Torcuato Di Tella. Pp. 97-144.

Кокот, R. 2005. Estructuras de defensa costera, $2^{a}$ Comunicación de Cambio Climático "vulnerabilidad de la zona costera". Fundación Torcuato Di Tella. Pp. 97-144.

Kutschker, A; C Brand \& ML Miserendino. 2009. Evaluación de la calidad de los bosques de ribera en ríos del NO del Chubut sometidos a distintos usos de la tierra. Ecol. Austral, 19(1):19-34.

Licursi, M; N Gómez \& J Donadelli. 2010. Ecological optima and tolerances of coastal benthic diatoms in a freshwater mixohaline zone from the Río de la Plata estuary. Mar. Ecol.-Prog. Ser., 418:105-117.

LÓPEZ, RA \& SC MARCOMINI. 2004. Análisis de los riesgos ambientales por antropogénesis y su valoración frente a los futuros cambios climáticos en el área metropolitana de la ciudad de Buenos Aires. Revista de la Asociación Geológica Argentina, 59(3):416-422.

MADDOCK I. 1999. The importance of physical habitat assessment for evaluating river health. Freshwater biology, 41:373-391.

Meng, L; CD Orphanides \& JC Powell. 2002. Use of a fish index to assess habitat quality in Narragansett Bay, Rhode Island. T. Am. Fish Soc., 131:731-742.

MunNé, A; N Prat; C Solá; N Bonada \& M Rieradevall. 2003. A simple field method for assessing the ecological quality of riparian habitat in rivers and streams: QBR index. Aquatic Conservation: Aquat. Conserv.: Mar. Freshwat. Ecosyst., 13:147-163.

RAAB, D \& SE BAYLEY. 2012. A vegetation-based Index of Biotic Integrity to assess marsh reclamation success in the Alberta oil sands, Canada. Ecol. Indic., 15:43-51.

Rodrigues Capítulo, A; M Tangorra \& CS Ocón. 2001. Use of Benthic macroinvertebrate to assess the ecological status of pampean rivers (Argentine). Aquat. Ecol., 35: 109-119.

Shannon, CE \& W WeAver. 1949. A Mathematical Theory of Communication. London: University Illinois Press.

SladeCeK, V. 1973. System of water quality from the biological point of view. Arch. Hydrobiol., 7:1-218.

Troitiño, E; MC Costa; L Ferrari \& A Giorgi. 2010. La conservación de las zonas ribereñas de un arroyo pampeano. I Congreso Internacional de Hidrología de Llanuras Azul, Buenos Aires, Argentina. Tomo II:777-784.

WINWARD, AH. 2000. Monitoring the vegetation resources in riparian areas. Gen. Tech. Rep. RMRSGTR-47. Ogden, UT: U.S. Department of Agriculture, Forest Service, Rocky Mountain Research Station. Pp. 49. 


\section{INFORMACIÓN SUPLEMENTARIA}

Tabla 1. Promedio y desvío estándar $(n=106)$ de las principales características físico-químicas del agua de los sitios de muestreo correspondientes al período 2005 -2008 en la Franja Costera Sur del Río de la Plata.

Table 1. Mean and standard deviation $(n=106)$ of the main physical-chemical characteristics in the water of the sampling sites during the study period (2005-2008) throughout the Franja Costera Sur of the Río de la Plata.

\begin{tabular}{|c|c|c|c|c|c|c|c|c|}
\hline Sitio & $\mathrm{pH}$ & $\begin{array}{l}\text { Conductividad } \\
(\mu \mathrm{S} / \mathrm{cm})\end{array}$ & $\begin{array}{c}\text { Oxígeno } \\
\text { Disuelto }(\mathrm{mg} / \mathrm{L})\end{array}$ & $\begin{array}{c}\mathrm{PO}_{4}^{-3} \\
(\mathrm{mg} / \mathrm{L})\end{array}$ & $\begin{array}{c}\mathrm{NO}_{3}^{-} \\
(\mathrm{mg} / \mathrm{L})\end{array}$ & $\begin{array}{c}\mathrm{NO}_{2}^{-} \\
(\mathrm{mg} / \mathrm{L})\end{array}$ & $\begin{array}{c}\mathrm{NH}_{4}^{+} \\
(\mathrm{mg} / \mathrm{L})\end{array}$ & $\begin{array}{c}\mathrm{DBO}_{5} \\
(\mathrm{mg} / \mathrm{L})\end{array}$ \\
\hline $\mathrm{DL}$ & $7.8( \pm 0.9)$ & $532( \pm 758)$ & $8.0( \pm 2.1)$ & $0.3( \pm 0.3)$ & $0.7( \pm 0.3)$ & $0.0( \pm 0)$ & $0.2( \pm 0.2)$ & $2.6( \pm 1.5)$ \\
\hline SI & $7.6( \pm 0.4)$ & $314( \pm 63)$ & $7.2( \pm 0.7)$ & $.2( \pm 0.1)$ & $1.1( \pm 0.2)$ & $0.1( \pm 0)$ & $0.3( \pm 0.1)$ & $3.8( \pm 2.2)$ \\
\hline $\mathrm{AE}$ & $7.7( \pm 0.6)$ & $618( \pm 954)$ & $6.4( \pm 1.2)$ & $0.9( \pm 0.4)$ & $0.9( \pm 0.4)$ & $0.1( \pm 0)$ & $0.3( \pm 0.1)$ & $3.4( \pm 1.8)$ \\
\hline cos & $8.0( \pm 0)$ & $346( \pm 0)$ & $7.4( \pm 0)$ & $0.1( \pm 0)$ & $1.4( \pm 0)$ & $0.1( \pm 0)$ & $0.2( \pm 0)$ & $14.7( \pm 0)$ \\
\hline $\mathrm{R}$ & $7.0( \pm 0)$ & $562( \pm 0)$ & $20(+0)$ & $0.3( \pm 0)$ & $0.3( \pm 0)$ & $0.0( \pm 0)$ & $2.4( \pm 0)$ & $15.0( \pm 0)$ \\
\hline CSA & $7.9( \pm 0.7)$ & $707( \pm 265)$ & $5.6( \pm 1.4)$ & $0.4( \pm 0.2)$ & $0.4( \pm 0.3)$ & $0.1( \pm 0.1)$ & $2.3( \pm 0.1)$ & $15.0( \pm 1.4)$ \\
\hline SD & $7.9( \pm 0.3)$ & $1058( \pm 559)$ & $2.6( \pm 2.3)$ & $1.5( \pm 1.1)$ & $1.0( \pm 1.5)$ & $0( \pm 0.1)$ & $1.7( \pm 1.0)$ & $12.7( \pm 1.2)$ \\
\hline $\mathrm{BE}$ & 8.2 & 561 & 3) & 5) & $0.7)$ & 1) & $( \pm 0.9)$ & $8.5( \pm 4.4)$ \\
\hline $\mathrm{BZ}$ & $8.4( \pm 0.4)$ & $467( \pm 92)$ & $9.1( \pm 2.7)$ & $0.3( \pm 0.1)$ & $1.2( \pm 0.3)$ & $0.2( \pm 0.1)$ & $0.6( \pm 0.6)$ & $6.6( \pm 4.0)$ \\
\hline $\mathrm{BC}$ & $8.5( \pm 0.7)$ & $347( \pm 72)$ & $9.2( \pm 1.6)$ & $0.2( \pm 0.1)$ & $1.0( \pm 0.4)$ & $0.0( \pm 0)$ & $0.1( \pm 0.1)$ & $5.3( \pm 4.3)$ \\
\hline PL & $8.5( \pm 0.7)$ & $323( \pm 110)$ & $10.3( \pm 3.0)$ & $0.3( \pm 0.2)$ & $1.1( \pm 0.5)$ & $0.0( \pm 0)$ & $0.2( \pm 0.2)$ & $7.5( \pm 5.0)$ \\
\hline PLP & $8.0( \pm 0)$ & $429( \pm 0)$ & $6.9( \pm 0)$ & $0.2( \pm 0)$ & $1.1( \pm 0)$ & $0.1( \pm 0)$ & $0.3( \pm 0)$ & $2.4( \pm 0)$ \\
\hline LBO & $8.3( \pm 0.4)$ & $550( \pm 88)$ & $9.5( \pm 0.5)$ & $0.3( \pm 0)$ & $0.7( \pm 0.5)$ & $0.1( \pm 0.1)$ & $1.2( \pm 1.1)$ & $5.2( \pm 1.1)$ \\
\hline BAG & $7.8( \pm 0.2)$ & $573( \pm 241)$ & $5.7( \pm 1.9)$ & $0.7( \pm 0.4)$ & $0.5( \pm 0.5)$ & $0.1( \pm 0.1)$ & $0.5( \pm 0.5)$ & $16.2( \pm 9.0)$ \\
\hline BAL & $8.5( \pm 0.6)$ & $410( \pm 145)$ & $9.3( \pm 1.5)$ & $0.1( \pm 0.1)$ & $0.4( \pm 0.4)$ & $0.0( \pm 0)$ & $0.0( \pm 0)$ & $6.7( \pm 2.2)$ \\
\hline EP & $8.4( \pm 0)$ & $564( \pm 0)$ & $11.1( \pm 0)$ & $0.1( \pm 0)$ & $0.1( \pm 0)$ & $0.0( \pm 0)$ & $0.1( \pm 0)$ & $3.2( \pm 0)$ \\
\hline $\mathrm{AT}$ & $8.2( \pm 0.2)$ & $732( \pm 452)$ & $8.6( \pm 0.5)$ & $0.1( \pm 0.1)$ & $0.4( \pm 0.3)$ & $0.0( \pm 0)$ & $0.3( \pm 0.5)$ & $7.9( \pm 3.8)$ \\
\hline M & $8.4( \pm 0.7)$ & $1299( \pm 1069)$ & $8.8( \pm 0.9)$ & $0.1( \pm 0.1)$ & $0.5( \pm 0.5)$ & $0.0( \pm 0)$ & $0.0( \pm 0)$ & $8.5( \pm 7.2)$ \\
\hline $\mathrm{P}$ & $8.6( \pm 0.5)$ & $1857( \pm 1430)$ & $9.1( \pm 0.7)$ & $0.1( \pm 0.1)$ & $0.5( \pm 0.5)$ & $0.0( \pm 0)$ & $0.1( \pm 0.1)$ & $5.2( \pm 4.8)$ \\
\hline SS & $8.0( \pm 0)$ & $5393( \pm 0)$ & $8.5( \pm 0)$ & $0.1( \pm 0)$ & $0.2( \pm 0)$ & $0.0( \pm 0)$ & $0.1( \pm 0)$ & $4.3( \pm 0)$ \\
\hline PI & $8.2( \pm 0.5)$ & $5313( \pm 4573)$ & $8.4( \pm 1.0)$ & $0.1( \pm 0.1)$ & $0.3( \pm 0.3)$ & $0.0( \pm 0)$ & $0.0( \pm 0)$ & $7.0( \pm 6.7)$ \\
\hline
\end{tabular}

